\title{
Non-technical skills for surgeons: challenges and opportunities for cardiothoracic surgery
}

\author{
Dominique Vervoort ${ }^{1}$, Sameer Hirji ${ }^{2}$ \\ ${ }^{1}$ Johns Hopkins Bloomberg School of Public Health, Baltimore, Maryland, USA; ${ }^{2}$ Division of Cardiac Surgery, Department of Surgery, Brigham and \\ Women's Hospital, Boston, Massachusetts, USA \\ Correspondence to: Dominique Vervoort, MD. Johns Hopkins Bloomberg School of Public Health, 615 North Wolfe Street, 21205 Baltimore, \\ Maryland, USA. Email: vervoortdominique@hotmail.com.
}

Submitted Jan 13, 2020. Accepted for publication Feb 07, 2020.

doi: $10.21037 /$ jtd.2020.02.16

View this article at: http://dx.doi.org/10.21037/jtd.2020.02.16

In their review, Casali et al. comprehensively describe the potential of non-technical skills for surgeons (NOTSS) in thoracic surgery (1). While illustrating the contemporary growing focus on patient safety mechanisms within the medical care framework, the authors conclude with an often overlooked yet vital component of surgical care: NOTSS. Patient outcomes have traditionally been associated with surgeons technical skills (how well does one operate), institutional volume (how much can one operate), and patient factors (how well does one rehabilitate/recover). Non-operative skills, or so-called "soft skills", have rarely been taken into consideration until a few years ago. It has become clearly evident that human factors play an integral role in influencing patient outcomes, responsible for up to $60 \%$ of surgery-related adverse events (2). NOTSS builds upon established frameworks of situation awareness, decision making, communication and teamwork, and leadership skills to enhance team communication in the operating room and, accordingly, reduce the occurrence of preventable adverse or 'failure to rescue' events (Figure 1). While the authors describe new avenues for NOTSS implementation in thoracic surgery, limited knowledge and implementation thereof exists within cardiothoracic surgery as a whole to date.

Few simulation models and NOTSS-like training modules have been developed and piloted in the United States, commonly with the end goal of improving communication and streamlining teamwork within the operating room during time-critical scenarios $(3,4)$. Notably, NOTSS training programs have demonstrated substantial promise and feasibility in improving trainees' leadership and communication skills during major surgery; however, longterm impact (i.e., whether or not these skills fade) remains unknown. These results raise a few important preliminary questions: (I) are our surgeons and residents adequately trained in leadership and communication? (II) To what extent do training programs around the world integrate these concepts as a mandatory component of cardiothoracic surgical training? (III) Are standardized curricula necessary and should they be adopted by regional and national accreditation bodies? (IV) Should NOTSS certification be introduced alongside other standardized testing for accreditation and obtaining licenses? It is clear that conservative measures do not correlate well with clinicians' future performance. For example, the United States Medical Licensing Examination (USMLE) Step 1 has shown poor correlation with medical students' clinical performance later in their careers, although may be associated with higher emotional intelligence $(5,6)$. Similarly, general surgery residents' performance on the American Board of Surgery In-Training Examination (ABSITE) seems to limitedly correlate with patient outcomes by these providers down the line (7).

NOTSS training programs, whether through simulation training or naturalistic surgical coaching, are increasingly integrated in non-cardiothoracic surgical specialties in both high-income and low- and middle-income country settings $(8,9)$. High-intensity specialties such as cardiothoracic surgery are especially prone to human factor variability which can impact patient outcomes. Thus, there ought to be widespread concerted efforts to integrate non-technical skills programs within the training curriculum and practice 


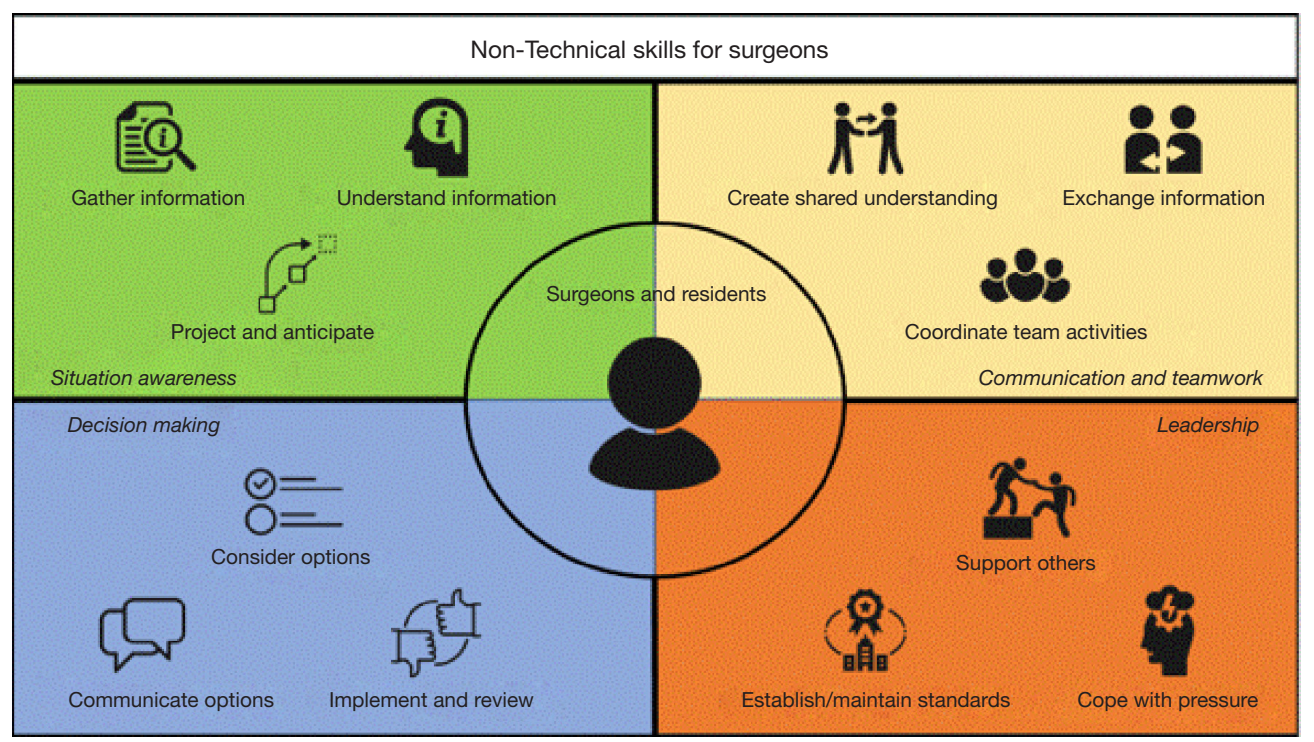

Figure 1 Non-technical skills for surgeons.

of modern cardiothoracic surgery. In an era in which patient safety and quality of care are so ingrained in academic medicine, and closely tied to hospital reimbursements and ratings systems (e.g., the Society of Thoracic Surgeons Star rating), professional societies should adopt such a focus similar to the integration of enhanced recovery after surgery (ERAS) models in clinical practice. We believe this is an opportune time for professional societies to take initiative, and spearhead efforts to adopt non-technical skills within its membership.

Ultimately, teaching non-technical skills is innately patient-centred or, perhaps better, patient-oriented, and can play an important role in reducing preventable errors, improve the care team's satisfaction, improve patient outcomes, reduce health care costs, and better distribute hospital resources. The cardiothoracic community ought to develop a holistic culture of patient safety and consider team communication in the operating room and across the patient care continuum a critical entity.

\section{Acknowledgments}

Funding: None.

\section{Footnote}

Conflicts of Interest: The authors have no conflicts of interest to declare.
Ethical Statement: The authors are accountable for all aspects of the work in ensuring that questions related to the accuracy or integrity of any part of the work are appropriately investigated and resolved.

Open Access Statement: This is an Open Access article distributed in accordance with the Creative Commons Attribution-NonCommercial-NoDerivs 4.0 International License (CC BY-NC-ND 4.0), which permits the noncommercial replication and distribution of the article with the strict proviso that no changes or edits are made and the original work is properly cited (including links to both the formal publication through the relevant DOI and the license). See: https://creativecommons.org/licenses/by-nc-nd/4.0/.

\section{References}

1. Casali G, Lock G, Novoa NM. Teaching non-technical skills: the patient centered approach. J Thorac Dis 2019. doi: 10.21037/jtd.2019.01.48.

2. Gillespie BM, Harbeck E, Kang E, et al. Effects of a Brief Team Training Program on Surgical Teams' Nontechnical Skills: An Interrupted Time-Series Study. J Patient Saf 2017. [Epub ahead of print].

3. Yule S, Gupta A, Gazarian D, et al. Construct and criterion validity testing of the Non-Technical Skills for Surgeons (NOTSS) behaviour assessment tool using videos of simulated operations. Br J Surg 2018;105:719-27. 
4. Bierer J, Memu E, Leeper WR, et al. Development of an In Situ Thoracic Surgery Crisis Simulation Focused on Nontechnical Skill Training. Ann Thorac Surg 2018;106:287-92.

5. Wagner JG, Schneberk T, Zobrist M, et al. What Predicts Performance? A Multicenter Study Examining the Association Between Resident Performance, Rank List Position, and United States Medical Licensing Examination Step 1 Scores. J Emerg Med 2017;52:332-40.

6. Hollis RH, Theiss LM, Gullick AA, et al. Emotional intelligence in surgery is associated with resident job satisfaction. J Surg Res 2017;209:178-83.

Cite this article as: Vervoort D, Hirji S. Non-technical skills for surgeons: challenges and opportunities for cardiothoracic surgery. J Thorac Dis 2020;12(3):1112-1114. doi: 10.21037/ jtd.2020.02.16
7. Ray JJ, Sznol JA, Teisch LF, et al. Association Between American Board of Surgery In-Training Examination Scores and Resident Performance. JAMA Surg 2016;151:26-31.

8. Pradarelli JC, Delisle M, Briggs A, et al. Identifying Naturalistic Coaching Behavior Among Practicing Surgeons in the Operating Room. Ann Surg 2019. [Epub ahead of print].

9. Lin Y, Scott JW, Yi S, et al. Improving Surgical Safety and Nontechnical Skills in Variable-Resource Contexts: A Novel Educational Curriculum. J Surg Educ 2018;75:1014-21. 